\title{
Sensor of Electric Field on the Basis of Nanoporous Alumina Oxide
}

\author{
Svetlana N. Kurilkina, Nikolai I.Mukhurov \\ B.I.Stepanov Institute of Physics of National Academy of Sciences of Belarus, 68 Nezavisimosti Ave., \\ 220072 Minsk, Belarus \\ s.kurilkina@ifanbel.bas-net.by
}

\begin{abstract}
:
The novel type has been proposed of sensor of electrical field, the operation of which is based on the phenomenon of birefringence in nanoporous alumina oxide with the pores filled by electro-sensitive material. Our investigations show technological performability of this high-sensitive and cheap sensor. The technical advantage of the sensor is the possibility of the use as sensitive substance of a number of nano-structured materials having electro-optical coefficients in several orders higher in comparison with ones for bulk materials. The proposed sensor on the basis of nanoporous alumina oxide is flexible, reconfigurable with the possibility of embedding in mobile manufacturing line.
\end{abstract}

Key words: nanoporous alumina oxide, electro-optic effect, electro-sensitive polymer.

\section{Physical Foundation of Electrical Sensor on the Basis of Nanoporous Alumina Oxide}

Oxide-aluminum technology attracted much attention as it enables mass production without the use of expensive lithographic tools, such as electron beam exposure system. As a rule, the layers of porous alumina are usually formed by electrochemical etching of aluminum foil substrates in various acids [1]. The resulting porous layer is a set of oxide cells in the center of which a hollow channel is located [2-6] (fig. 1). The distance between the pores becomes larger linearly with increasing the voltage. Pore diameter is determined by the rate of chemical dissolution of aluminum oxide, depending on the used electrolyte, and can widely vary (from $10 \mathrm{~nm}$ to 5 microns). Al-substrate can be absent in the final sample of nanoporous alumina oxide.
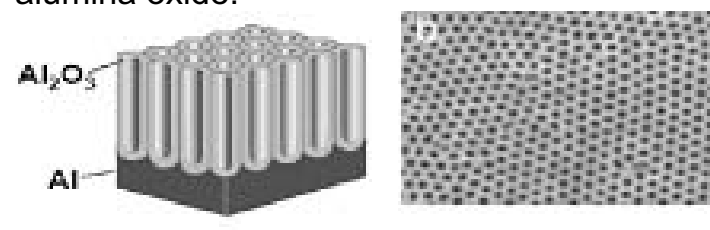

Fig. 1. Structure of matrix of nanoporous alumina oxide.

If the typical pore sizes are much smaller than the wavelength of incident light, this nano- structured dielectric can be studied as a uniform optically uniaxial medium having two main efficient refraction indices $n_{e f s, p}=\sqrt{\varepsilon_{e f s, p}}$ determining according to Bruggeman model [7]: $\left(\frac{\varepsilon_{1}-\varepsilon_{e f s, p}}{\varepsilon_{e f s, p}+L\left(\varepsilon_{1}-\varepsilon_{e f s, p}\right)}\right)(1-f)+\left(\frac{\varepsilon_{2}-\varepsilon_{e f s, p}}{\varepsilon_{e f s, p}+L\left(\varepsilon_{2}-\varepsilon_{e f s, p}\right)}\right) f=0$

Here $\varepsilon_{1}, \varepsilon_{2}$ are dielectric constants of $\mathrm{Al}_{2} \mathrm{O}_{3}$ and pore filler substance, respectively; $f$ is the porosity (volumetric part of pore in material); $L$ is the factor of depolarization. Note that for the case of cylindrical pores $L=0$ (or $L=0.5$ ) if electrical vector of propagating wave is directed along (perpendicular to) the pore axis.

Let external medium, having the refraction index $n$ (for example, air), be bounded with nanoporous material (fig.2). Then, due to Brewster effect if the condition is fulfilled

$$
n \operatorname{tg} \theta_{B}=n_{e f p}
$$

the p-polarized component with electric vector in the plane of incidence is absent in the reflected light. Note that Brewster angle $\theta_{B}$ 
depends on the optical constant of pore filler substance and porosity.

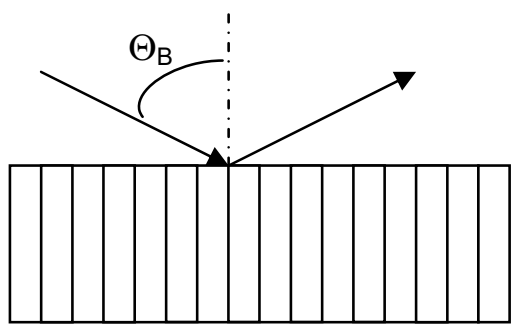

Fig. 2. Scheme of incidence of light beam on the matrix of nanoporous alumina oxide.

Let now the pores of matrix of alumina oxide be filled by electro-sensitive isotropic substance with dielectric permittivity

$$
\varepsilon_{2_{i j}}\left(E_{0}\right)=\varepsilon_{2} \delta_{i j}-\varepsilon_{2}^{2} R_{i j k l} E_{0_{k}} E_{0_{l}},
$$

which depends on the external electrical field $E_{0}$. Here $\varepsilon_{2}$ is the dielectric constant of the pore filler substance in the absence of external field, $\delta_{i j}$ is the unit tensor, $R_{i j k l}=R_{\mu \nu}$ is the tensor of electro-optic coefficients of Kerr. Then, in the presence of external electric field the pore filler medium becomes uniaxial with optical axis along the applied field.

As follows from [8], if the light beam propagates in $\vec{n}$ direction at the angle $\varphi$ to $\vec{E}_{0}$, the refraction coefficients for the waves with electric vector orthogonal and parallel the plane $\left(\vec{n} \vec{E}_{0}\right)$ $\left(n_{2}^{o}\left(E_{0}\right)\right.$ and $n_{2}^{e}\left(E_{0}\right)$, respectively) are determined by relations:

$$
\begin{aligned}
& n_{2}^{o}\left(E_{0}\right)=\sqrt{\varepsilon_{2}}-\frac{1}{2} \varepsilon_{2}^{3 / 2} R_{12} E_{0}^{2}, \\
& n_{2}^{e}\left(E_{0}\right)=\sqrt{\varepsilon_{2}}-\frac{1}{2} \varepsilon_{2}^{3 / 2}\left[R_{12}+\Delta R \sin ^{2} \varphi\right] E_{0}^{2} .
\end{aligned}
$$

Here $\quad \Delta R=R_{11}-R_{12}, \quad R_{11,12} \quad$ are scalar electro-optic constants characterizing quadratic electro-optic effect in isotropic medium.

As follows from Eq.(1), the efficient refraction indices of the porous medium filled by electrosensitive isotropic substance depend on $E_{0}$. Owing to this, the Brewster angle is changed and in the presence of external electric field it can be presented as

$$
\theta_{B_{E}}=\theta_{B}+\Delta \theta_{B},
$$

where $\Delta \theta_{B}$ is a small value. Then knowing the value of the angle variations $\Delta \theta_{B}$, from Eqs. (2), (1), taking into account the values of electric-optical coefficients of a pore filler substance, it is possible to estimate the value of the field influencing.

Note that for registration of the Brewster angle variation the goniometer can be used which gives the opportunity to measure the angle $\Delta \theta_{B}$ with error $\sim 2$ '.

This is the physical foundation of electrical sensor on the basis of nanoporous alumina oxide.

\section{Estimation of Sensitivity of Electrical Sensor on the Basis of Nanoporous Alumina Oxide}

As an electro-sensitive medium one can use polymer-stabilized liquid crystal (PSLC) which shows large electro-optic effect. According to paper [9] the Kerr constant

$$
K=\Delta n \lambda E_{0}^{2}=\frac{1}{2} \varepsilon_{2}^{3 / 2} \Delta R \sin ^{2} \varphi E_{0}^{2}
$$

in this material is equal to $7.2 \cdot 10^{-9} \mathrm{~m} / \mathrm{V}^{2}$. It is essentially more than the Kerr constant for the widely used nitrobenzene and other isotropic liquids. Estimations show that using polymerstabilized liquid crystal as a filler of alumina oxide porous matrix one can register the electric strength $E_{0} \sim 10^{2} \mathrm{~V} / \mathrm{m}$. Taking into account that the thickness of matrix can be up to $1 \mu \mathrm{m}$, we conclude that this element gives the opportunity to estimate electric field up to $10^{-4} \mathrm{~V}$.

The application of CdSe/PDDA polymer nanoclusters with electro-optic constants $R_{11}=2 \cdot 10^{-8} \mathrm{~m}^{2} / \mathrm{V}^{2}, R_{12}=7 \cdot 10^{-9} \mathrm{~m}^{2} / \mathrm{V}^{2}$

[10] as a filler for porous alumina oxide gives the opportunity to enhance the sensitivity of the sensor up to $10^{-5} \mathrm{~V}$.

As follows from Eqs. (1), (2), (4), the value of the registered voltage is determined by relation:

$$
U=\left[\frac{2 \Delta \theta_{B}\left(1+\varepsilon_{e f p}\right)}{\varepsilon_{2}^{3 / 2}\left[R_{12}+\Delta R \sin ^{2} \varphi\right]}\right]^{1 / 2} D,(7)
$$

where $D$ is the thickness of alumina oxide matrix,

$$
\begin{aligned}
& \varepsilon_{e f p}=-\frac{\left(\varepsilon_{2}-\varepsilon_{1}\right)(1-2 f)}{2}+ \\
& +\sqrt{\left[\frac{\left(\varepsilon_{2}-\varepsilon_{1}\right)(1-2 f)}{2}\right]^{2}+\varepsilon_{1} \varepsilon_{2} .}
\end{aligned}
$$

It is clear from Eqs. (7), (8) that the sensitivity of the proposed sensor $\left(U_{\lim }=U\left(\Delta \theta_{B}=2^{\prime}\right)\right)$ depends on optical constants of porous matrix and filled substance and on the porosity. These dependences are illustrated in figs.3,4. 


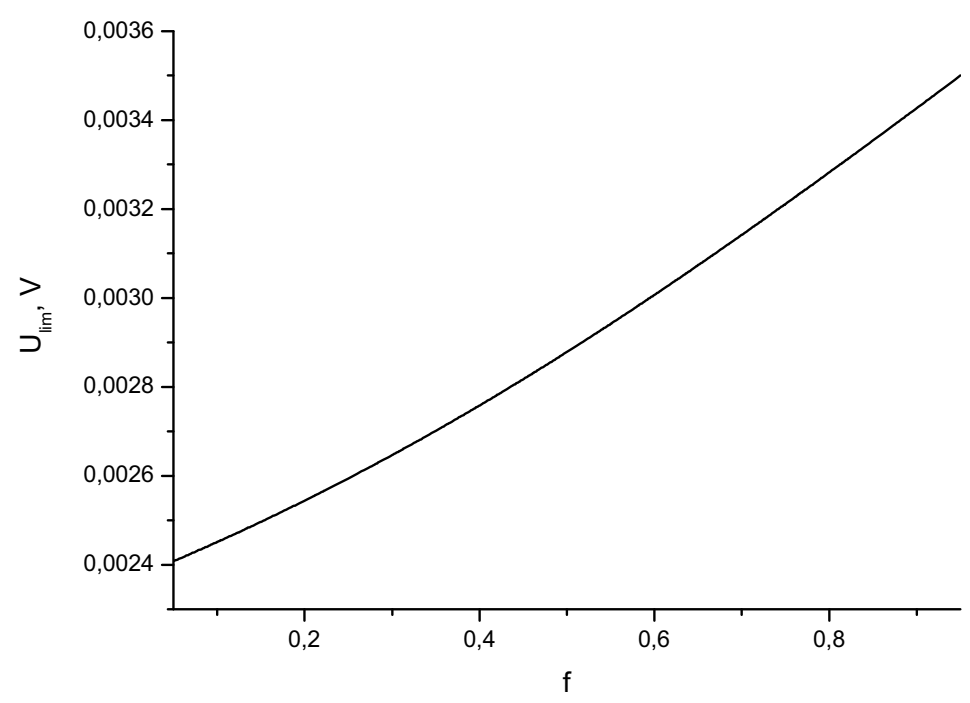

Fig.3. The dependence of sensitivity of the proposed sensor on the porosity. Dielectric permeability of porous alumina oxide is 1.58. The thickness of porous matrix is $10 \mu \mathrm{m}$. The filler substance is CdSe/PDDA nano-clusters. The wavelength is $632.8 \mathrm{~nm}$

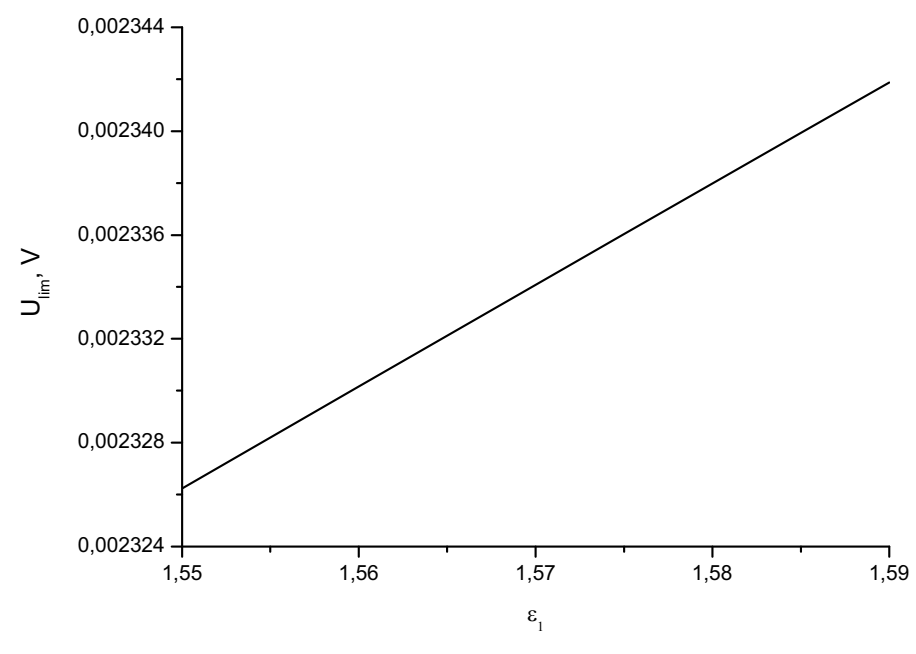

Fig.4. The dependence of sensitivity of the proposed sensor on the dielectric permeability of porous matrix. The porosity is 0.3 . The thickness of porous matrix is $10 \mu \mathrm{m}$. The filler substance is

CdSe/PDDA nano-clusters. The wavelength is $632.8 \mathrm{~nm}$.

As follows from figs.3,4, the sensitivity of the proposed sensor decreases with increasing the porosity and/or dielectric permeability of the porous substance.

\section{Conclusion}

Thus, in the paper the novel type has been proposed of electrical sensor, the operation of which is based on the phenomenon of induced birefringence in nanoporous alumina oxide with the pores filled by electro-sensitive material. This cheap sensor gives the opportunity of use as sensitive substance of a number of nanostructured materials having electro-optical coefficients in several orders higher in comparison with ones for bulk materials. So, it allows one to achieve the sensitivity essentially higher than one for the known sensors. As the investigations show the proposed sensor on the basis of nanoporous alumina oxide is flexible, reconfigurable with the possibility of embedding in mobile manufacturing line. 


\section{References}

[1] L.A. Golovan', V.Yu.Timoshenko, P.K.Kashkarov, Phyzics - Uspekhi 177, 619-638 (2007); doi: 10.3367/UFNr.0177.200706b.0619

[2] H. Masuda, L. Yada, A. Osaka, Jpn.J.Appl.Phys. 37, L1340-L1342 (1998); doi: 10.1143/JJAP.37.L1340

[3] O. Jessensky, F. Müller, U. Gösele, Appl.Phys.Lett. 72, 1173-1175 (1998); doi: $\underline{10.1063 / 1.121004}$

[4] J. De Laet, H. Terryn, J.Vereecken, Thin Solid Films 320 241-250 (1998); doi: 10.1016/S00406090(97)00741-4

[5] K Yasui et al, J. Vac. Sci.Tech. B23, L9 (4 pages) (2005); doi:10.1116/1.1941247

[6] H. Masuda et al, Jpn.J.Appl.Phys. 38, L1403 L1405 (1999); doi: 10.1143/JJAP.38.L1403

[7] J.E. Spanier, I.P. Herman, Phys. Rev. B61, 10437-10450 (2000)

[8] A. Yariv, P.Yeh, Optical waves in crystals. New York-Chichester-Brisbane-Toronto-Singapore: A Wiley Interscience Publication, 1987. 616 p.

[9] Y. - C. Yang, D. - K. Yang, Appl. Phys. Lett. 98, 023502-1 - 023502-3 (2011); doi: $10.1063 / 1.3533396$

[10] F. Zhang, Electro-optic Properties of Semiconductor Nano-crystals And Electro-optic Polymers And Their Applications [Electronic resources]/ Mode of access: http://scholar.lib.vt.edu/theses/available/etd10172002-101754/ 